\title{
Directed Transfer Function is not influenced by volume conduction-inexpedient pre-processing should be avoided
}

\author{
Maciej Kaminski* and Katarzyna J. Blinowska \\ Department of Biomedical Physics, Faculty of Physics, University of Warsaw, Warsaw, Poland \\ ${ }^{*}$ Correspondence: maciek.kaminski@fuw.edu.pl \\ Edited by: \\ Hava T. Siegelmann, Rutgers University, USA \\ Reviewed by: \\ Coralie Ebert, Weizmann Institute of Science, Israel
}

Keywords: Directed Transfer Function, volume conduction, multivariate methods of connectivity estimation, pre-processing

The problem of brain connectivity has been gaining more and more interest in the last years. Connectivity can be estimated by different techniques and at the different levels of the hierarchy of the nervous system. Here we shall consider functional connectivity at the level of the brain structures, in particular connectivity measures derived from scalp EEG measurements. A multitude of estimators are being used for connectivity estimation: linear, nonlinear, bivariate and multivariate. The disadvantage of bivariate estimators is connected with the fact, that in virtue of common feeding many spurious connections are found. This fact was demonstrated in works: (Blinowska et al., 2004; Kuś et al., 2004). Let us consider the common situation when a source is emitting activity measured at $N$ electrodes. In case of bivariate measures beside $N$ true connections also false connections between all electrodes will be find in virtue of common feeding and their number will be $[N(N-$ 1) $/ 2-N]$, so they will outnumber the true connections.

This fact directed the interest of the scientific community to the multivariate methods of connectivity estimation. In particular, multivariate estimators based on the Granger causality principle allow to estimate directed connectivity and does not produce spurious connections. Among these estimators widely applied are: Directed Transfer Function (Kamiński and Blinowska, 1991) and Partial Directed Coherence (Baccala and Sameshima, 2001) based on the Multivariate Autoregressive Model (MVAR). Currently DTF and PDC are commonly used and have became a part of various signal processing packages, e.g., eConnectome (http://econnectome.umn.edu), Octave-Forge (http://octave.sourceforge. net/tsa function mvfreqz.html), Epilab (http://www.epilepsiae.eu/project_outputs/ epilab_software), SIFT (http://sccn.ucsd. edu/wiki/SIFT).

Unfortunately we have noticed that quite often the application to DTF of inappropriate preprocessing routines produces misleading results. Moreover, the use of these routines is not necessary in virtue of the properties of DTF. As the authors of the DTF estimator, we are particularly concerned that the method is applied correctly.

Among the pre-processing methods used before DTF application the most common approach is to project the signals into the source space in order to eliminate volume conduction effect. However, DTF for a pair of channels $i$ and $j$ is nonzero only, if there is a phase difference between channels $i$ and $j$.

The volume conduction is a propagation of the electromagnetic field, so it does not produce a phase difference at electrodes. Therefore, DTF is practically insensitive to volume conduction. The fact that the estimators of connectivity based on the phase difference between channels are not influenced by the volume conduction was also recognized in Stam et al. (2009). The influence of a signal of a constant phase on DTF was demonstrated by means of simulation. For the set of EEG signals we have added a sinusoid, with the same phase for each signal. The amplitude of that sinusoid (of $20 \mathrm{~Hz}$ ) was similar to the amplitudes of EEG signals. The result is shown in Figure 1. We can observe a prominent peak at $20 \mathrm{~Hz}$ in the power spectra of the signals, but this peak is absent in DTF functions.

The fact that DTF is not influenced by volume conduction effects is further supported by the excellent topographical agreement of DTF results with the evidence obtained from anatomical data, imaging studies and physiological experiments. Clear cut patterns of propagation emerged from these studies. As the examples may serve the studies of finger movement (Ginter et al., 2001; Kuś et al., 2006), localization of epileptic focus (Franaszczuk et al., 1994) results obtained for Constant Attention Test (Blinowska et al., 2010) and working memory paradigm (Brzezicka et al., 2010; Blinowska et al., 2013). More examples may be found in Blinowska (2011); Blinowska and Zygierewicz (2011). The animations illustrating dynamically changing patterns of connectivity obtained by the Short-time Directed Transfer Function (SDTF) in some of the above mentioned experiments are available at http://brain.fuw.edu.pl/ kjbli.

Since DTF is immune to volume conduction the pre-processing procedures such as projections on the cortex surface or Laplace transform are not needed. Moreover, they destroy the original correlation structure of the set of signals. If we mix the information from different channels calculating for example Laplacian, we influence the correlation between signals and the information about the phase relations between channels is disturbed. 


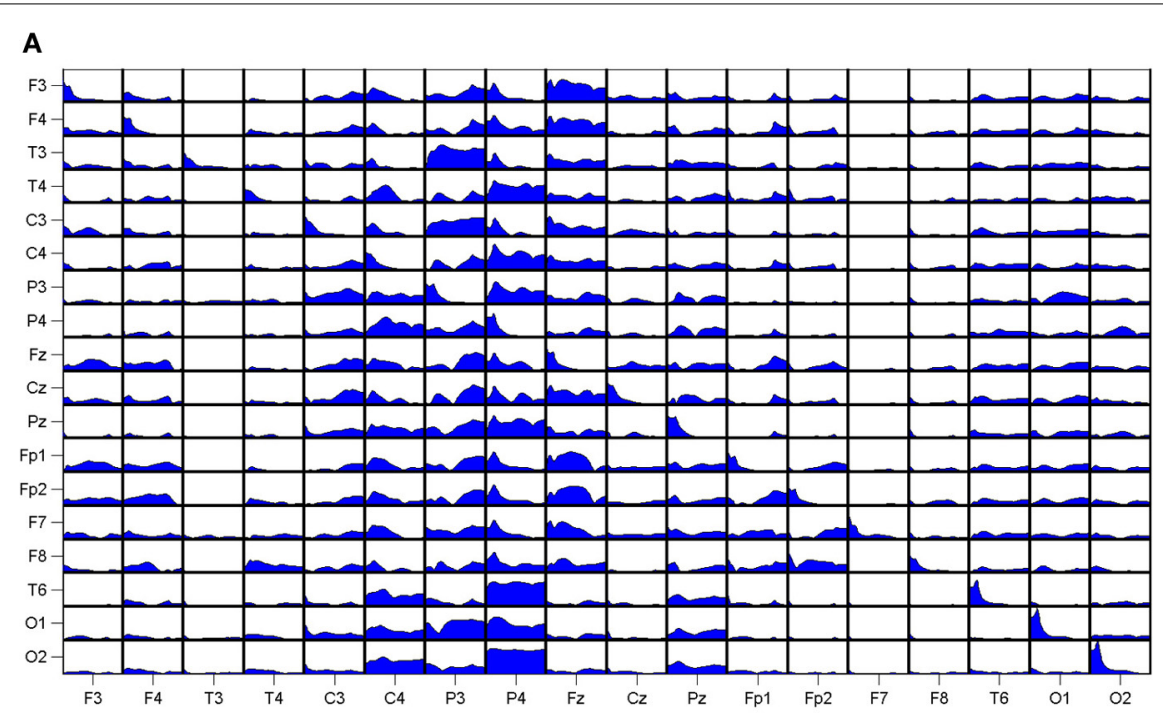

B

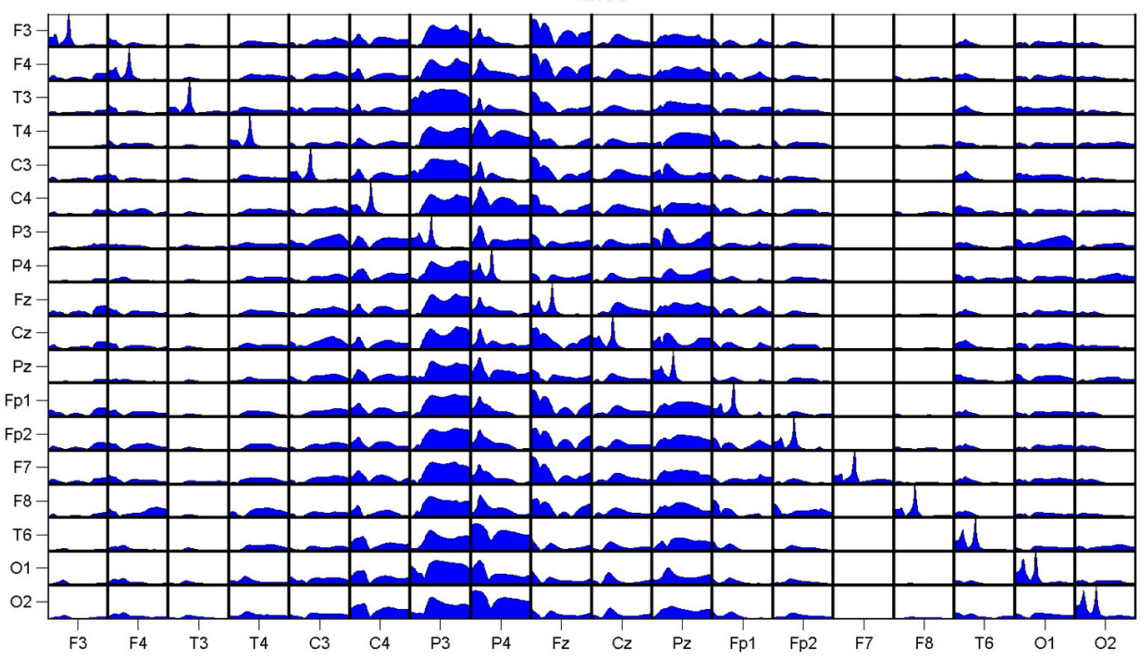

C

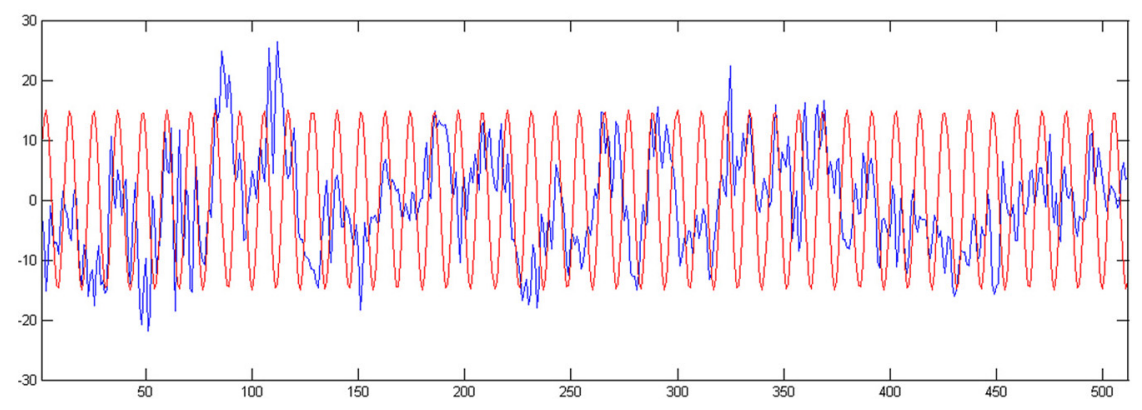

FIGURE 1 | The DTFs (as functions of frequency) estimated for a set of EEG signals. (A) The results for original dataset, (B) same data with $20 \mathrm{~Hz}$ sinusoid added with constant phase to each channel. On the diagonals of the

panels power spectra are shown. The propagation from the channel marked below the pictures to the channel marked at the left. At the (C) EEG from one channel with superimposed sinusoid.

As a result, information on causality or, in another words, on the propagation of activity from channel $j$ to $i$ is lost.

The results of the works where the preprocessing involving projection on the cortex was applied show a disorganized structure of connectivity. The preprocessing of the data before the DTF application should be limited to subtraction of the mean, possibly division by the variance and digital filtering. However, the filtering must not influence phases of signals. These can be achieved by filtering forward and backward (e.g., Matlab procedure filtfilt). The signals should be 
referenced to the "neutral" derivation, e.g., linked ears, nose, or similar. No common average or bipolar reference may be used. There should be no preprocessing by means Hjorth or Laplace transform, Independent Components Analysis or projection of signals on the cortex. In the view of robustness of DTF in respect to volume conduction, these procedures are obsolete, furthermore, they are harmful and may produce misleading results.

It is worth mentioning that in general methods of connectivity estimation based on phase differences are insensitive to volume conduction. For instance this property holds also for Partial Directed Coherence, but not for ordinary coherence, because it contains common component, which includes activity of no phase difference between electrodes. In conclusion we would like to underline that the preprocessing of the signals for estimation of connectivity should be based on the thorough understanding of the properties of the applied methods.

\section{ACKNOWLEDGMENT}

The work was supported by the Statutory Grant of Polish Ministry of Science and Higher Education to Faculty of Physics of Univeristy of Warsaw.

\section{REFERENCES}

Baccala, L. A., and Sameshima, K. (2001). Partial directed coherence: a new conception in neural structure determination. Biol. Cybern. 84 , 463-474. doi: 10.1007/PL00007990

Blinowska, K. J. (2011). Review of the methods of determination of directed connectivity from multichannel data. Med. Biol. Engin. Comput. 49, 521-529. doi: 10.1007/s11517-011-0739-x

Blinowska, K. J., Kaminski, M., Brzezicka, A., and Kaminski, J. (2013). Application of directed transfer function and network formalism for assessment of functional connectivity in working memory task. Philos. Trans. A Math. Phys. Eng. Sci. 371:20110614. doi: 10.1098/rsta.2011.0614

Blinowska, K. J., Kuś, R., and Kamiński, M. (2004). Granger causality and information flow in multivariate processes. Phys. Rev. E 70:050902. doi: 10.1103/PhysRevE.70.050902

Blinowska, K. J., Kuś, R., Kaminski, M., and Janiszewska, J. (2010). Transmission of brain activity during cognitive task. Brain Topogr. 23, 205-213. doi: 10.1007/s10548-010-0137-y

Blinowska, K. J., and Zygierewicz, J. (2011). Practical Biomedical Signal Analysis Using Matlab. Boca Raton; London; New York: CRC Press.

Brzezicka, A., Kaminski, M., Kaminski, J., and Blinowska, K. J. (2010). Information transfer during transitive reasoning task. Brain Topogr. 24, 1-8. doi: 10.1007/s10548-010-0158-6

Franaszczuk, P. J., Bergey, G. J., and Kamiński, M. (1994). Analysis of mesial temporal seizure onset and propagation using the directed transfer function method. Electroenceph. Clin Neurophysiol. 91, 413-427. doi: 10.1016/0013-4694(94)9 0163-5

Ginter, J. Jr., Blinowska, K. J., Kaminski, M., and Durka, P. J. (2001). Phase and amplitude analysis in time-frequency space-application to voluntary finger movement. J. Neurosci. Methods 110 , 13-124. doi: 10.1016/S0165-0270(01)00424-1

Kamiński, M., and Blinowska, K. J. (1991). A new method of the description of the information flow in brain structures. Biol Cybern. 65, 203-210. doi: 10.1007/BF00198091
Kuś, R., Ginter, J. Jr., and Blinowska, K. J. (2006). Propagation of EEG activity during finger movement and its imagination. Acta Neurobiol. Exp. 66, 195-206.

Kuś, R., Kamiński, M., and Blinowska, K. J. (2004). Determination of EEG activity propagation: pair-wise versus multichannel estimate. IEEE. Trans. Biomed. Eng. 51, 1501-1510. doi: 10.1109/TBME.2004 827929

Stam, C. J., de Haan, W., Daffertshofer, A., Jones, B. F., Manshanden, I., van Cappellen van Walsum, A. M., et al. (2009). Graph theoretical analysis of magnetoencephalographic functional connectivity in Alazheimer disease. Brain 132, 213-224. doi: 10.1093/brain/ awn 262

Conflict of Interest Statement: The authors declare that the research was conducted in the absence of any commercial or financial relationships that could be construed as a potential conflict of interest.

Received: 03 September 2013; accepted: 20 May 2014; published online: 10 June 2014.

Citation: KaminskiM and Blinowska KJ (2014) Directed Transfer Function is not influenced by volume conduction-inexpedient pre-processing should be avoided. Front. Comput. Neurosci. 8:61. doi: 10.3389/ fncom.2014.00061

This article was submitted to the journal Frontiers in Computational Neuroscience.

Copyright (c) 2014 Kaminski and Blinowska. This is an open-access article distributed under the terms of the Creative Commons Attribution License (CC BY). The use, distribution or reproduction in other forums is permitted, provided the original author(s) or licensor are credited and that the original publication in this journal is cited, in accordance with accepted academic practice. No use, distribution or reproduction is permitted which does not comply with these terms. 